\title{
Some answers, some questions
}

$\mathrm{R}$ ecent decades have seen a great deal of progress in the prevention, diagnosis and therapy of venous thromboembolism. However, our surgical brethren have done much better in the important area of prevention than have we medical types. For decades, it has been clear that perioperative anticoagulation in a wide variety of patients undergoing surgery is of benefit in terms of avoiding postoperative venous thrombosis and its dangerous consequence - pulmonary embolism. It is not clear why similar data were slow to accumulate for medical patients who should be at similar risk. Perhaps it was because medical patients, as a heterogeneous group, do not lend themselves to the kind of crisp definition that is the case in people with fractured hips, for example. Perhaps it was because medical patients are less uniformly immobilized for a standard period of time, and perhaps it was because pulmonary embolism can be extremely difficult to diagnose in patients with substantial pre-existing lung disease, as is often the case with medical patients. In any event, routine anticoagulation therapy was not a standard of care in medical patients for at least a decade after it became routine in many surgical patients.

This situation has changed, or should have changed. The recent Prophylaxis in Medical Patients with Enoxaparin (MEDENOX) trial (1) comparing the effects of two doses of low molecular weight heparin (enoxaparin) with placebo in medical patients has shown a clear benefit with $40 \mathrm{mg}$ of enoxaparin a day in preventing venous thrombosis. The study should be of interest to the readers of the Canadian Respiratory Journal for two reasons. First, Canadian investigators and patients were major contributors to the trial. Second, and more important, the patients studied were the type of patients that we care for - those with respiratory failure and severe infections, plus another risk factor for thromboembolic disease such as age or obesity. Patients were treated for the duration of their hospital admission or for six to 14 days. The incidence of venous thrombosis and/or pulmonary emboli was reduced by almost two-thirds with enoxaparin during the first two weeks, and was reduced by more than one-half over three months. The message seems clear such patients should be treated with low molecular weight heparin.

In the present issue of the Canadian Respiratory Journal, we publish an economic analysis of the MEDENOX trial (pages 169-177). In my experience, it is an unusually clear exposition of an important problem. The costs of medical care were enumerated for a Canadian patient population with the same outcomes in the first two weeks of therapy, but the analysis was extended to cover three months. Threemonth data from the MEDENOX trial could

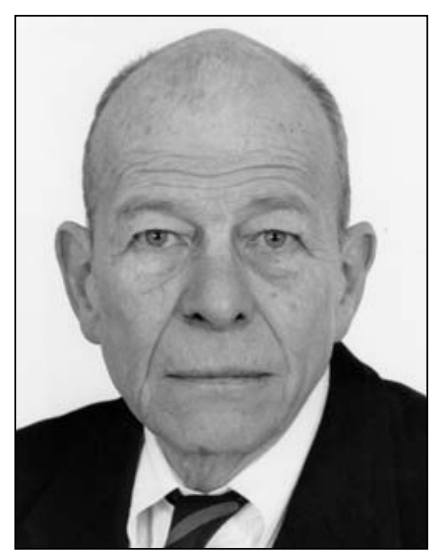

Nick R Anthonisen not be used because trial patients who were found to have thromboembolic disease were treated appropriately, though they were largely asymptomatic. Thus, the natural history of the disease was changed after the first two weeks, and the extension of the analysis to three months dealt with likely outcomes in patients who had thromboembolic disease at two weeks. Obviously, there were fewer of these patients in the enoxaparin group than in the untreated group, but costs per patient had been higher due to the costs associated with the drug. Subsequent costs were measured assuming that $35 \%$ of proximal deep venous thrombosis would become symptomatic and require therapy, while this would be true of $5 \%$ of distal thromboses. Costs were assessed as a function of whether the patients were treated at a tertiary centre, where only $10 \%$ of them would be admitted to hospital, or a community hospital, where the admission rate was $50 \%$. In the former setting, where outpatient care was the rule, there were fewer events in the enoxaparin group but costs per event were higher, so that it cost $\$ 87$ to avoid one thromboembolic event, which the authors judged appropriately to be cost effective. In the model with a substantial readmission rate for symptomatic venous thrombosis, enoxaparin reduced cost by virtue of reduced hospital admissions. The authors point out that in reality, a $10 \%$ readmission rate for symptomatic venous thrombosis is probably low, even in tertiary centres, and that the higher the readmission rate, the more economical enoxaparin becomes. The authors performed a sensitivity analysis, and concluded that their 
findings were robust, even when the assumed rates of symptom development were changed substantially.

There remain a couple of questions. The first is whether more prolonged treatment would have been better, particularly in avoiding events that occurred after two weeks. This can only be answered by a subsequent trial. The second is the issue of bleeding. Though there was no significant difference between enoxaparin and placebo in the frequency of major bleeds, and therefore no cost differences assessed, it is difficult (at least for me) to believe that even low dose therapy with low molecular weight heparin will not be associated with some hemorrhagic complications. However, the MEDENOX trial was carried out under field conditions; that is, anticoagulant administration was not associated with other special procedures. Therefore, I believe we can assume that bleeding complications are unlikely to be common in the real world.

\section{Nick R Anthonisen MD Editor-in-Chief Canadian Respiratory Journal}

\section{REFERENCE}

1. Samama MM, Cohen AT, Darmon J-Y, et al. A comparison of enoxaparin with placebo for the prevention of venous thromboembolism in acutely ill medical patients. Prophylaxis in Medical Patients with Enoxaparin Study Group. N Engl J Med 1999;341:793-800. 


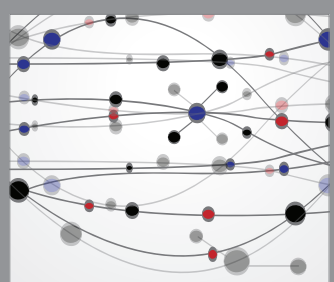

The Scientific World Journal
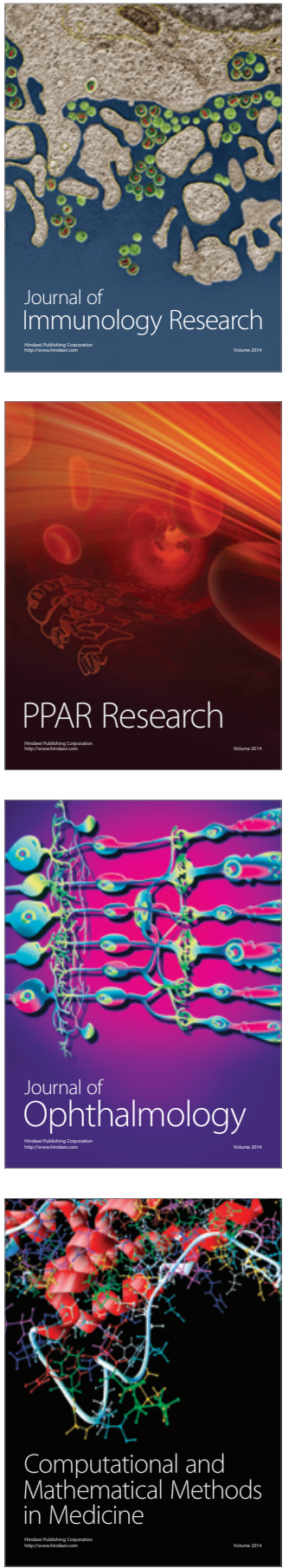

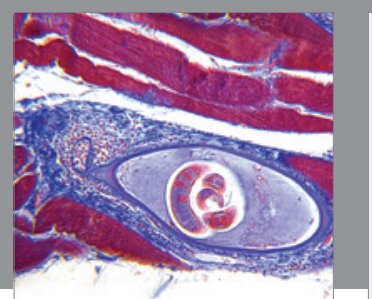

Gastroenterology Research and Practice

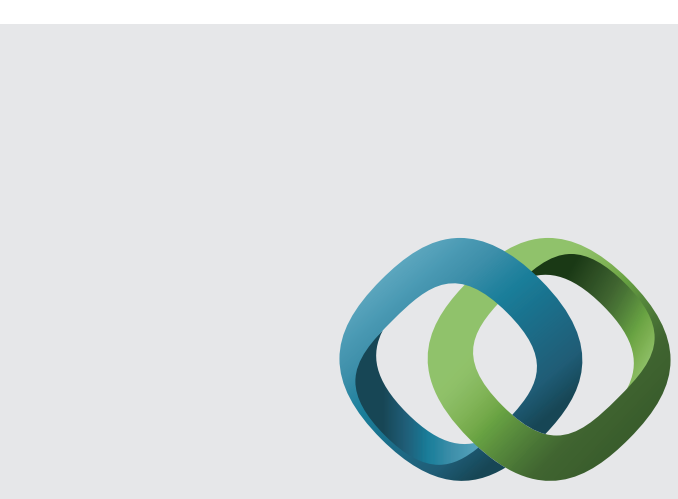

\section{Hindawi}

Submit your manuscripts at

http://www.hindawi.com
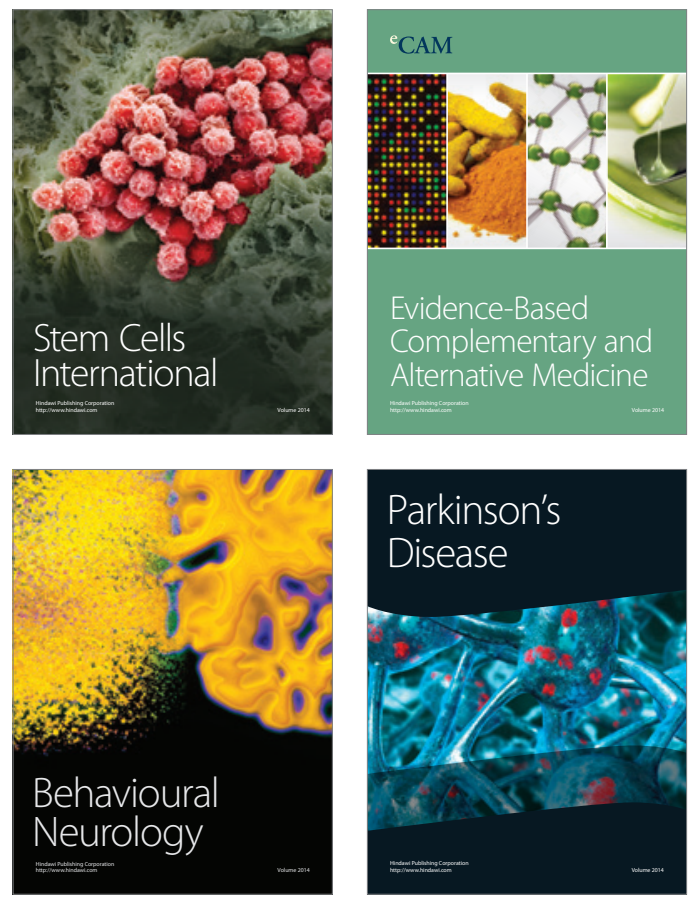
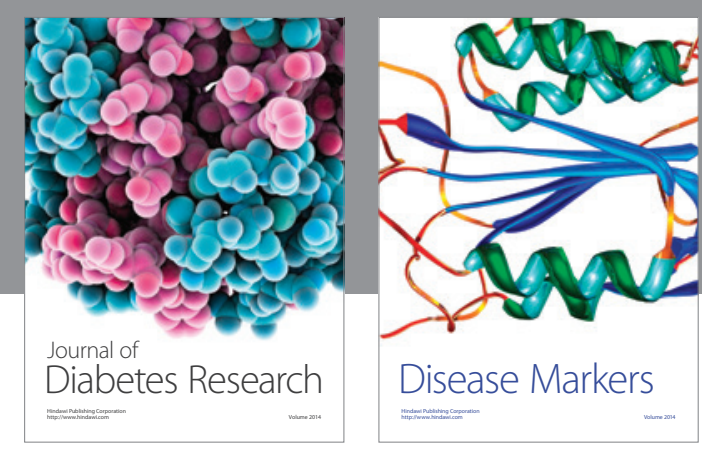

Disease Markers
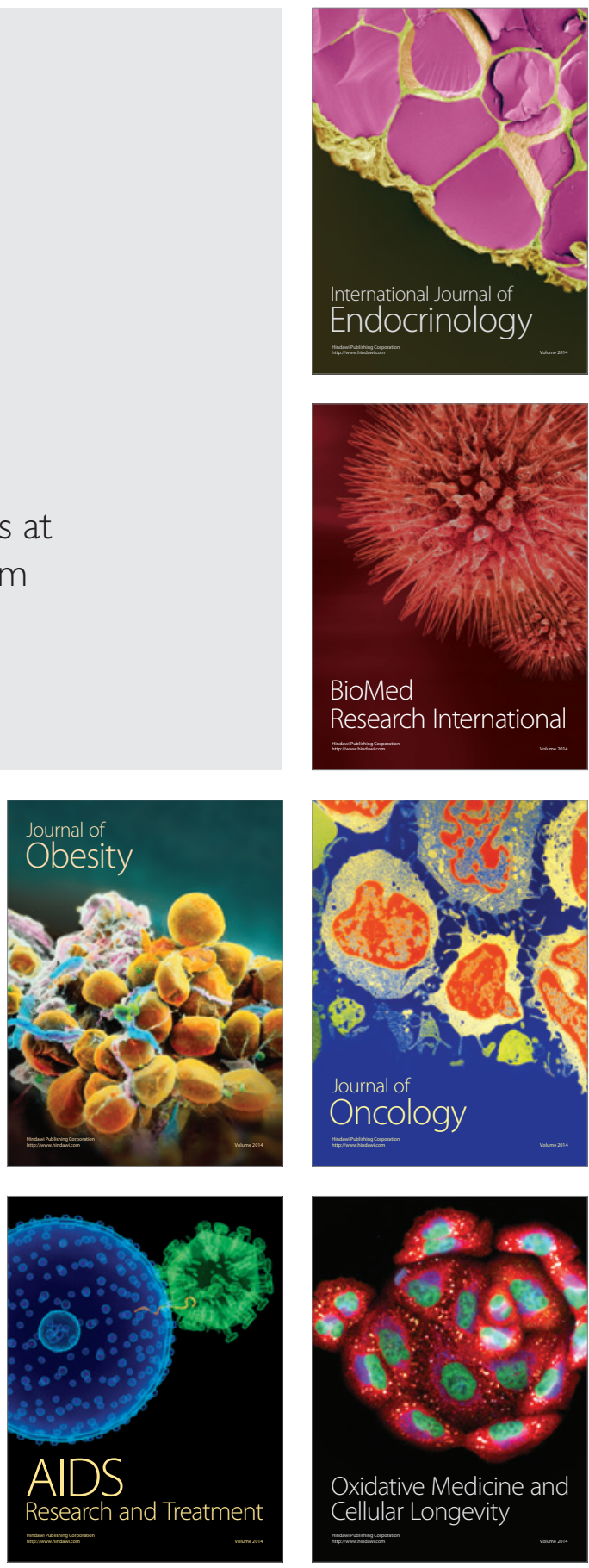